\title{
Erratum
}

\section{Approximate Normalized Wave Functions for the Finite-Size Coulomb Problem}

\author{
J.L. Friar \\ Medium Energy Physics Theory Group, Los Alamos Scientific Laboratory, \\ University of California, Los Alamos, New York Mexico, USA \\ Z. Phys. A - Atoms and Nuclei 292, 1 (1979)
}

Section III contains several errors. A typographical error occurs in the bracketted quantity in (38), which should read $\left(2 / t^{2}+1 / t^{4}\right)$. Equation (42) contains a serious error in the second term. It should read

$$
\begin{aligned}
& f(x)=\frac{4}{35 x}+\frac{2 x}{15}-\frac{1}{3 x} \int_{1}^{\infty} \frac{d u}{u^{4}}\left(u^{2}-1\right)^{\frac{1}{2}}\left(2 / u^{2}+1 / u^{4}\right) e^{-x u} \\
& \cong \frac{5 \pi}{96}+\frac{\pi x^{2}}{32} .
\end{aligned}
$$

Equation (43) then reads

$$
\begin{aligned}
& \Delta E_{V P}^{e}=-\frac{4 Z \alpha^{2}\left|\phi_{n}(0)\right|^{2}}{15 m^{2}} \\
& \cdot\left(1-\frac{15 \pi}{16} Z \alpha \mu m\left\langle r^{2}\right\rangle_{(2)}-\frac{25 Z \alpha \mu \pi}{64 m}+\ldots\right)
\end{aligned}
$$

Neglecting finite size in the wave functions alone would replace $\left\langle r^{2}\right\rangle_{(2)}$ by $\left\langle r^{2}\right\rangle$. Furthermore, $\left\langle r^{2}\right\rangle_{(2)} \equiv 2\left\langle r^{2}\right\rangle$ for spherical distributions. Thus, the electronic vacuum polarization finite size correction for electronic atoms is of relative order $Z \alpha m^{2}\left\langle r^{2}\right\rangle$, neglecting reduced mass effects. This is quite tiny for $H$, since $m^{2}\left\langle r^{2}\right\rangle$ is smaller than $\alpha^{2}$.

The author would like to thank Don Yennie for pointing out the error.

\section{J.L. Friar}

Medium Energy Physics Theory Group

Los Alamos Scientific Laboratory

University of California

Los Alamos, NM 87545

USA 